An ectopic pregnancy occurs in approximately $0.5-2 \%$ of all pregnancies. The number of cases of cervical cancer in women under 30 years of age in Poland is less than 100 cases per year. The case presented herein concerns a rare clinical situation of a 29-year-old woman admitted to the Gynaecological Oncology Department with a diagnosis of cervical cancer. The main symptom resulting in the patient's referral was ascites. The presence of free fluid in the peritoneal cavity in patients diagnosed with a malignant tumour suggests a high degree of progression of the underlying disease, though this could not be confirmed in a clinical study in this case. An interview, examination, and laboratory tests confirmed a coexisting life-threatening ruptured tubal pregnancy. The consequences of not recognising both an ectopic pregnancy and cervical cancer in a patient presenting with nonspecific clinical symptoms could pose a serious threat to health and life.

Key words: ectopic pregnancy, cervical cancer, ascites.

Contemp Oncol (Pozn) 2021; 25 (4): 295-297 DOI: https://doi.org/10.5114/wo.2021.112166

\section{Coexistence of a ruptured ectopic pregnancy and cervical cancer: how to avoid a diagnostic error when the same symptoms present two different diagnoses}

\author{
Kamila Kaźmierczak ${ }^{1}$, Witold Cholewiński ${ }^{2}$, Błażej Nowakowski ${ }^{1}$
}

${ }^{1}$ Surgical, Oncological and Endoscopic Gynaecology Department, Greater Poland Cancer Centre, Poznań, Poland

${ }^{2}$ Nuclear Medicine Unit, Greater Poland Cancer Centre, Poznań, Poland

\section{Introduction}

A pregnancy is defined as ectopic when it is confirmed to be anywhere outside of the uterine cavity. An estimated $2 \%$ of all pregnancies are ectopic [1]. In 90\% of ectopic pregnancies, the egg implants in a fallopian tube [2]. The most important risk factors for ectopic pregnancy are the use of assisted reproductive techniques in cases of infertility, previous pelvic operations, pelvic inflammatory disease, smoking, and an age of over 35. Approximately half of all women with ectopic pregnancies do not have any known risk factors [3]. Despite the benefits of early diagnosis - mainly related to the ability to immediately measure the level of $\beta$-human chorionic gonadotropin $(\beta$-hCG) and to use transvaginal ultrasound - a ruptured ectopic pregnancy is still a significant cause of pregnancy-related morbidity and mortality (approximately $3.4 \%$ of pregnancy-related deaths in developed countries) [4]. The symptoms of ectopic pregnancy are nonspecific; abdominal pain, amenorrhea and vaginal bleeding are the most common. The initial diagnosis is based on the confirmation of pregnancy (by testing for the presence of $\beta$-hCG in the urine or blood serum) and performing an ultrasound scan, preferably transvaginal [5]. As a result of new advances in diagnostics, if diagnosed early on, an ectopic pregnancy can be treated conservatively with methotrexate or minimally invasive surgery techniques. However, some patients are admitted to the hospital in an unstable condition with bleeding in the peritoneal cavity, and there is a need for urgent surgical intervention [6]. The most important risk factor for developing cervical cancer is infection with oncogenic types of human papillomavirus. Factors contributing to human papillomavirus infection are multiple births, the use of oral contraceptives by women who simultaneously smoke, early sexual initiation, frequent changes in sexual partners, a positive history of sexually transmitted diseases, some autoimmune diseases, and chronic immunosuppression [7]. In the early stages of cervical cancer, clinical symptoms are absent or non-specific. The patient may present various kinds of abnormal bleeding (intermenstrual, contact or postmenopausal). In the treatment of cervical cancer, surgery, radiotherapy or chemotherapy are used in combination with surgery or radiotherapy, depending on the tumour stage [8]. According to the American Cancer Society, the 5-year survival rate in cervical cancer diagnosed at an early stage (limited to the uterus; FIGO stage I) is $92 \%$.

\section{Case report}

A 29-year-old patient was referred to the Gynaecological Oncology Department due to a diagnosis of cervical cancer accompanied by ascites. The 
referring physician issued an urgent referral due to a concern about the patient's general condition and the severity of lower abdominal pain. The patient had been taking oral contraception for 2.5 months, which was initiated by the attending gynaecologist due to menstrual disorders, abnormal spotting and intermenstrual bleeding. The patient had never been pregnant before and did not suffer from any chronic diseases. The removal of a throat cyst was the only surgery that the patient had undergone. Samples had been taken from the cervix a month earlier due to an abnormal Pap smear test result (Pap Smear IIIB); the histology results confirmed the glandular type of cervical cancer.

A few days before admission, the patient was hospitalised at the neurology department due to a brief loss of consciousness. The patient also complained of lower abdominal pain during her hospitalisation.

The general condition of the patient on admission to the Gynaecological Oncology Department was fair. In the physical examination, the predominant symptoms were pallor of the integuments and mucosa and tenderness with positive peritoneal symptoms in the lower abdomen. The patient's general condition did not correspond to the stage of cancer progression. The gynaecological examination revealed an ulcerated cervical tumour in the vaginal space between 6 and 9 o'clock, passing to the posterior vaginal fornix, as well as bleeding from the cervix. The enlargement and tenderness in the right appendages attracted attention. There was no infiltration of the parametrium. Transvaginal ultrasound revealed a hypoechoic cervical tumour $(30 \times 27 \mathrm{~mm})$ with a visible transition to the posterior vaginal vault and the body of the uterus, which was normal. Ovaries of normal size and morphology were visualized on both sides. The presence of a corpus luteum in the right ovary was suggested. Other abnormalities include the presence of free fluid in the pelvis and, on further examination, free fluid accumulation around the liver and spleen. Additionally, there was an irregular $5-\mathrm{cm}$ tumour near the right ovary, which could have corresponded to an ectopic pregnancy. Laboratory tests showed significant anaemia and a serum $\beta$-hCG concentration of $498 \mathrm{mlU} / \mathrm{ml}$, which clearly confirmed the assumption of a ruptured ectopic pregnancy. Due to the life-threatening condition, the patient underwent laparoscopic surgery $4 \mathrm{~h}$ after diagnosis. In the first step, $500 \mathrm{ml}$ of liquid blood was drained from the peritoneal cavity, then the distended, partially ruptured right fallopian tube was removed. The patient was given 2 units of red blood cell concentrate and was discharged home in a good condition after two days of hospitalisation. The patient received a recommendation of a routine postoperative inspection and then re-admission to treat cervical cancer. Three weeks after the laparoscopic surgery of the right tubal ectopic pregnancy, the patient underwent a radical Piver III hysterectomy and pelvic lymphadenectomy up to the height of the common iliac bifurcation. The operation and the postoperative course were uneventful.

The postoperative histopathology revealed stage II A1 cervical cancer, according to the FIGO 2009 classification. No metastasis was found in any of the 32 removed lymph nodes.

\section{Discussion}

The main symptoms reported by the patient were $a b$ normal bleeding, which in gynaecology is non-specific and can occur in both an ectopic pregnancy and cervical cancer, as well as in other gynaecological disorders. These two diagnoses are uncommon in everyday practice, but the coincidence of both in one patient is extremely rare. In the case in question, it should be noted that the failure to recognise either of these diseases could have resulted in life-threatening consequences. According to the documentation, the patient was treated without excluding significant diagnoses that could have posed a serious threat to health and life.

Oral contraception is often used by specialists in the case of irregular menstruation, which should not preclude careful laboratory and clinical diagnosis. Cervical cancer was diagnosed based on the result from Pap smear screening, although the gynaecological examination showed a macroscopically visible tumour. At the same time, the patient developed symptoms related to complications of the fallopian pregnancy in the form of rupture or miscarriage. Unfortunately, in the opinion of the doctor, these symptoms were associated with the cervical cancer. Pregnancy was not considered due to the use of contraception, which was carelessly administered for abnormal bleeding that could have resulted from either an ectopic pregnancy or cervical cancer. On admission to hospital, there was a discrepancy between the local tumour progression and the referral symptom of ascites. By extending the diagnostic tests, the initial assessment of the cervical cancer stage and the coexistence of a clinical emergency - a ruptured ectopic pregnancy requiring urgent surgery - were made possible. If the patient had been correctly diagnosed from the beginning, the ectopic pregnancy could have been avoided. In this case, the ruptured ectopic pregnancy was an additional unnecessary risk factor, both direct and oncological. The clinical situation presented herein proves that when starting treatment, the diagnosis and possible concomitant diagnoses should always be verified.

The authors declare no conflict of interest.

\section{References}

1. Barnhart KT, Franasiak J. ACOG Practice Bulletin No. 193: Tubal ectopic pregnancy. Obstet Gynecol 2018; 131: 91-103.

2. Bouyer J, Coste J, Fernandez H, Pouly JL, Job-Spira N. Sites of ectopic pregnancy: a 10 year population-based study of 1800 cases. Hum Reprod 2002; 17: 3224-3230.

3. Barnhart KT, Sammel MD, Gracia CR, Chittams J, Hummel AC, Shaunik $A$. Risk factors for ectopic pregnancy in women with symptomatic first-trimester pregnancies. Steril 2006; 86: 36-43.

4. Knafel A, Basta P, Skotniczny K, et al. Ectopic pregnancy rupture can it be prevented? Ginekol Pol 2009; 80: 734-739.

5. Bray F, Ferlay J, Soerjomataram I, et al. Global cancer statistics 2018 GLOBOCAN estimates of incidence and mortality worldwide for 36 cancer in 185 countries. CA Cancer J Clin 2018; 68: 394-424.

6. Wojciechowska U, Dzidkowska J, Tarkowski W. Nowotwory złośliwe w Polsce w 2002 roku. Centrum Onkologii - Instytut, Warszawa 2004. 
7. Tjalma WA, Fiander A, Reich O, et al. HERACLES/SCALE Study Group: differences in human papillomavirus type distribution in high-grade cervical intraepithelial neoplasia and invasive cervical cancer in Europe. Int I Cancer 2013; 132: 854-867.

8. Jach R, Sznurkowski JJ, Bidziński M, et al. Zalecenia Polskiego Towarzystwa Ginekologii Onkologicznej dotyczące diagnostyki i leczenia raka szyjki macicy. Curr Gynecol Oncol 2017; 15: 24-33.

\section{Address for correspondence}

\section{Kamila Kaźmierczak}

Surgical, Oncological and Endoscopic Gynaecology Department Greater Poland Cancer Centre

15 Garbary St.

61-866 Poznań, Poland

e-mail: kamilka35@icloud.com

Submitted: 04.11 .2021

Accepted: 03.12.2021 